\title{
Combined Used of Rheology and LF-NMR for the Characterization of PVP-Alginates Gels Containing Liposomes
}

Fanesi, Giulia; Abrami, Michela; Zecchin, Francesca; Giassi, Irina; Ferro, Elena Dal; Boisen, Anja; Grassi, Gabriele; Bertoncin, Paolo; Grassi, Mario; Marizza, Paolo

Published in:

Pharmaceutical Research

Link to article, DOI:

$10.1007 / \mathrm{s} 11095-018-2427-0$

Publication date:

2018

Document Version

Publisher's PDF, also known as Version of record

Link back to DTU Orbit

Citation $(A P A)$ :

Fanesi, G., Abrami, M., Zecchin, F., Giassi, I., Ferro, E. D., Boisen, A., Grassi, G., Bertoncin, P., Grassi, M., \& Marizza, P. (2018). Combined Used of Rheology and LF-NMR for the Characterization of PVP-Alginates Gels Containing Liposomes. Pharmaceutical Research, 35(9), 1-11. https://doi.org/10.1007/s11095-018-2427-0

\section{General rights}

Copyright and moral rights for the publications made accessible in the public portal are retained by the authors and/or other copyright owners and it is a condition of accessing publications that users recognise and abide by the legal requirements associated with these rights.

- Users may download and print one copy of any publication from the public portal for the purpose of private study or research.

- You may not further distribute the material or use it for any profit-making activity or commercial gain

- You may freely distribute the URL identifying the publication in the public portal 


\title{
Combined Used of Rheology and LF-NMR for the Characterization of PVP-Alginates Gels Containing Liposomes
}

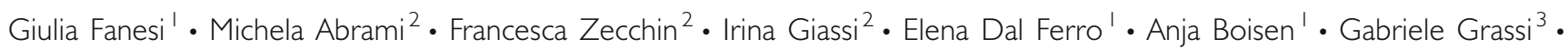 \\ Paolo Bertoncin ${ }^{4} \cdot$ Mario Grassi ${ }^{2}$ (D) Paolo Marizza'
}

Received: 31 January 2018 / Accepted: 7 May 2018

(C) Springer Science+Business Media, LLC, part of Springer Nature 2018

\begin{abstract}
Purpose This paper is based on the characterization of the rheological and Low Field NMR (LF-NMR) properties of an interpenetrated hydrogel made up by poly(N-vinyl-2-pyrrolidone) and sodium alginate. The final aim is to use the hydrogel as a delivery matrix for liposomes, widely used tools in the drug delivery field.
\end{abstract}

Methods Rheology, LF-NMR, TEM, cryo-TEM, confocal laser scanning microscopy and release test were employed to characterize the interpenetrated hydrogel. Different theoretical approaches such as Flory, Chui, Scherer and Schurz theories were used to interpret the experimental results.

Results We found that the crosslinking mechanisms of the two polymers produced an anti-synergistic effect on the final mechanical properties of the interpenetrated hydrogel. Instead of creating a continuous network, alginate formed isolated, cross-linked, clusters embedded in a continuous network of poly(N-vinyl-2-pyrrolidone). Additionally, gel structure significantly influenced liposome delivery.

Guest Editors: Kin Yip Tam, Zoran Mandic, and Tonglei Li

Electronic supplementary material The online version of this article (https://doi.org/I 0. I007/s I | 095-0 | 8-2427-0) contains supplementary material, which is available to authorized users.

Mario Grassi

mario.grassi@dia.units.it

Department of Micro- and Nanotechnology, Technical University of Denmark (DTU), Ørsteds Plads Bygning 345 Ø, 2800 Kgs, Kongens Lyngby, Denmark

2 Department of Engineering and Architecture, University of Trieste, Building B, via Valerio 6, I-34I 27 Trieste, Italy

3 Department of Life Sciences, Cattinara Hospital, University of Trieste, Strada di Fiume 447, I-34I 00 Trieste, Italy

4 Department of Life Sciences, University of Trieste, Piazzale Europa I, I-34I 27 Trieste, Italy
Conclusions The rheological and LF-NMR characterization were confirmed and supported by the independent techniques TEM, cryo-TEM and release tests Thus, our findings reiterate the potentiality of both rheology and LF-NMR for the characterisation of soft materials such as interpenetrated polymeric networks.

KEY WORDS Gel · LF-NMR · liposomes delivery . rheology · TEM

$\begin{array}{ll}\text { ABBREVIATIONS } \\ \text { CFM } & \text { Confocal fluorescence } \\ \text { DDS } & \text { Drug delivery system } \\ \text { DLS } & \text { Dynamic Light Scattering } \\ \text { DMPC } & \text { 1,2-dimyristoyl-sn-glycero-3-phosphocholine } \\ \text { DPPC } & \text { 1,2-dipalmitoyl-sn-glycero-3-phosphocholine } \\ \text { FS } & \text { Frequency sweep test } \\ \text { GTA } & \text { Glutaraldehyde } \\ \text { IPN } & \text { Interpenetrated network } \\ \text { LF-NMR } & \text { Low Field Nuclear Magnetic Resonance } \\ \text { LSM } & \text { Laser Scanning Microscope } \\ \text { NBD PE } & \text { 1,2-dioleoyl-sn-glycero-3- } \\ & \text { phosphoethanolamine-N-(7-nitro-2-1,3- } \\ & \text { benzoxadiazol-4-yl) } \\ \text { PVP } & \text { Poly(N-vinyl-2-pyrrolidone) } \\ \text { SS } & \text { Stress sweep test } \\ \text { TEM } & \text { Transmission electron microscopy }\end{array}$

\section{INTRODUCTION}

In the last two decades, many advances have been made in the pharmaceutical research with regard to the development of novel drug delivery system (DDS) [1]. Recently great attention has been focused on hydrogel-based DDS. Hydrogels consist of hydrophilic polymeric networks capable of imbibing a large 
amount of water molecules; moreover they exhibit an intrinsic low cytotoxicity and high biocompatibility. For these reasons, polymeric hydrogels are able to mimic soft living tissues much better than any other materials do. Hydrogels can be prepared by crosslinking of both natural and fully synthetic hydrophilic polymers. The crosslinking reaction converts the solution into a semi-ordered three-dimensional network, where the chains are connected to each other. According to the gelation mechanism, hydrogels are classified as either physical or chemical [2]. Chemical crosslinks are created by radical polymerization of low molecular weight monomers [3], reaction of complementary groups [4, 5], addition and condensation reactions [6], enzymatic reactions like that used to functionalize PEG with glutaminyl groups [7]. A particularly interesting approach employed for crosslinking is based on the exposure of the hydrogel to radiation. Gamma radiations have been utilized to trigger the crosslinking of polymers like chitosan/ poly(vinyl alcohol) (PVA) [8], PAA [9], PEG, PVP, agar based hydrogels [10], PCL [11] and polyurethanes (PCL-PEO) [12]. As an alternative to radiations, ultraviolet light (UV) has been proposed because of the more practical and less expensive nature. UV radiation activates a radical mechanism by means of a photoinitiator [13] whose reactivity determines the reaction rate, the spectral sensitivity (wavelength of absorption), the light resistance and the stability of the materials under storage conditions [14]. In this regard, the UV induced photocrosslinking of poly(vinyl pyrrolidone) (PVP) with hydrogen peroxide was earlier studied $[15,16]$. In a previous work, we deepened the study about PVP crosslinking by investigating the relation between different parameters such as photoinitiators, polymer concentrations, and exposure dose and the crosslinking of the hydrogel network [17]. By combining rotational rheology and low field nuclear magnetic resonance spectroscopy (LF-NMR), we could describe the structural properties of PVP hydrogels. We identified networks with a narrow mesh size distribution around $100 \mathrm{~nm}$. In the present study, we describe the synthesis of PVP-Alginate interpenetrated polymer networks (PVP-Alg IPNs). The attempt is to assess the effect of the sodium alginate addition to the PVP network structure recurring to the consolidated method of rheology and NMR spectroscopy. To evaluate the release properties of PVP-Alg IPNs, we incorporated in the polymer blend a suspension of fluorescently labelled liposomes whose dimensions were specifically chosen to be close to the estimated polymer mesh (nanometer range). In order to minimize the effect of charge interactions on the diffusion of nano-vesicles through the PVP-alginate IPN network, zwitterionic liposomes were prepared. In addition, to mimic drugladen liposomes and to have a reliable fluorescence signal, fluorescently-labelled liposomes were used.

Liposomes have been undertaken as they represent one of the most commonly employed delivery material for conventional and innovative nucleic acid based drugs [18-21].
Fluorescent microscopy images allowed observing liposomes distribution within the network at the different preparation stages of the hydrogel. Transmission Electron Microscopy (TEM) imaging provided a deeper understating of the hydrogel structure as well as of the liposomes after incorporation. Finally, the results of liposome release test provided further support to the structural interpretation and showed the performance of PVP-Alg IPNs as drug delivery system.

\section{MATERIALS AND METHODS}

\section{Materials}

The lipids used in the preparation of fluorescent liposomes were 1,2-dimyristoyl-sn-glycero-3-phosphocholine (DMPG) 1,2dipalmitoyl-sn-glycero-3-phosphocholine (DPPC), 1,2dioleoyl-sn-glycero-3-phosphoethanolamine-N-(7-nitro-2-1, 3-benzoxadiazol-4-yl) (NBD PE) $\left(\lambda_{\mathrm{abs}}=460 \mathrm{~nm}, \lambda_{\mathrm{em}}=\right.$ $535 \mathrm{~nm}$ ) all supplied by Avanti Polar Lipids. Poly(N-vinyl-2pyrrolidone) $\left(\mathrm{PVP} \sim 3.6 \cdot 10^{5} \mathrm{Da}\right)$, hydrogen peroxide $(30 \%$ wt) and sodium alginate $\left(\sim 10^{6} \mathrm{Da}\right.$; high $\alpha$-L-guluronic acid content $\sim 70 \%$ ) were provided by Sigma-Aldrich.

\section{Preparation of Liposomes and PVP-Alginate Blend Solutions}

The aqueous buffer Hepes2 was prepared by adding $10 \mathrm{mM}$ of 4-(2-hydroxyethyl)piperazine-1-ethane-sulfonic acid (HEPES) and $150 \mathrm{mM}$ of sodium chloride (NaCl) in Milli-Q water. The $\mathrm{pH}$ of the obtained solution was measured and adjusted to the value of 7.4. Liposomes were prepared by the thin-film hydration method followed by extrusion: lipid powder was dissolved in chloroform $\left(2.5 \mathrm{mg} \mathrm{mL}^{-1}\right)$ in single vials. In particular, $1 \mathrm{ml}$ of solution, contain $2.1 \mathrm{mg} \mathrm{DMPC}$ and $0.4 \mathrm{mg}$ DPPC lipids. In the preparation of fluorescentlylabelled liposomes, $20 \mu \mathrm{L}$ of NBD PE solution $\left(1 \mathrm{mg} \mathrm{mL}^{-1}\right)$ was added to the lipids mixture. Dry nitrogen was insufflated into the vial to let chloroform evaporation, thus forming a thin yellowish lipid film on the vial surface. Subsequently, vials were connected to a vacuum pump for $1 \mathrm{~h}$ to remove the residual organic solvent in the films. The dry lipids films were then suspended in Hepes 2 at $37^{\circ} \mathrm{C}$ under gentle agitation. The resulting multi-lamellar vesicles suspensions were submitted to extrusion at $37^{\circ} \mathrm{C}$ for 21 passages through polycarbonate membrane with $50 \mathrm{~nm}$ pores (Avanti Mini Extruder). For each liposome batch, Dynamic Light Scattering and $\zeta$ potential analysis (NanoBrook ZetaPlus, Brookhaven Instruments, USA) were evaluated to monitor the liposome size distribution and charges. Afterwards, fluorescently labelled dispersions were stored at $4^{\circ} \mathrm{C}$ inside amber vials until further use. Subsequently, polymer solutions of PVP and PVP with Alginate were prepared by dissolving the powders in 
mixtures of Hepes 2 (10 mM HEPES and $150 \mathrm{mM} \mathrm{NaCl}$ in Milli-Q water, resulting in $98 \% \mathrm{v} / \mathrm{v})$ to which $\mathrm{H}_{2} \mathrm{O}_{2}(2 \% \mathrm{v} / \mathrm{v})$ was added under $250 \mathrm{rpm}$ stirring at $50^{\circ} \mathrm{C}$ until a homogeneous mixing was achieved. Solutions were prepared fixing PVP and alginate concentration at 20 and $0.5 \%$ wt, respectively (PVP-A).

For the preparation of liposome laden hydrogels, the Hepes 2 was replaced with liposome suspension $(75 \% \mathrm{v} / \mathrm{v}$ $\left.2.5 \mathrm{mg} \mathrm{mL}^{-1}\right)$. After preparation, polymer solutions were poured into syringes and left for $24 \mathrm{~h}$ at room temperature for bubbles removal.

\section{Preparation of PVP-Alginate Blend Gels}

PVP -Alginate hydrogels preparation followed a sequential method. Three groups of samples were prepared: semi-IPNs hydrogels in which only PVP (system $a$ ) or Alginate (system $b$ ) were crosslinked, and a full IPN where both polymers have been crosslinked sequentially (system $c$ ). In order to get PVP crosslinking, about $1 \mathrm{~mL}$ of polymer solution was cast in cylindrical moulds ( $\sim 1 \mathrm{~mm}$ in depth, $\sim 35 \mathrm{~mm}$ in diameter). The moulds were placed at $15 \mathrm{~cm}$ from the UV source and irradiated for $33 \mathrm{~min}$ in a UV light chamber (BS-02, Dr. Gröbel UV Elektronik, Germany) equipped with 4 tubes emitting in the UVB range (265-400 $\mathrm{nm}$ ) and 4 tubes UVC range (single peak at $253.4 \mathrm{~nm}$ ). As the emitted power density was equal to $29 \mathrm{~mW} / \mathrm{cm}^{2}$, each mould received an irradiated energy equal to $100 \mathrm{~J}$. In order to prepare the full-IPN, following UV irradiation, defined volumes of a $\mathrm{CaCl}_{2}$ aqueous solution $\left(\left[\mathrm{Ca}^{++}\right]=9 \mathrm{~g} / \mathrm{l}\right)$ were sprayed onto the surface of gels specimens placed in a Petri dish and allowed to react for $5 \mathrm{~min}$. The crosslinked alginate IPNs were prepared pouring the polymer blend solution in a $1 \mathrm{~mm}$ thick film inside a Petri dish, and spraying the $\mathrm{CaCl}_{2}$ solution as above described.

\section{Rheology}

The rheological characterization of hydrogels was carried out at $25.0^{\circ} \mathrm{C}$ and $37^{\circ} \mathrm{C}$ using a controlled-stress rheometer RS150 (ThermoHaake, Germany) equipped with parallel plates (PP20Ti, diameter $20 \mathrm{~mm}$ ) having serrated surfaces, to avoid possible wall slippage, and provided with a Haake-F6 thermostat for temperature control. The measuring device was kept inside a glass bell at saturated humidity conditions to avoid evaporation effects. Hydrogels samples $\quad(\sim 1 \mathrm{~mm}$ thick) were removed with the aid of a small spatula from the Petri dish in which they were prepared and subsequently put on a wood surface in order to cut a cylinder of $20 \mathrm{~mm}$ diameter. Then, the cylinder was placed on the lower plate of the measuring device. The upper plate was then lowered to get in contact with hydrogel surface. Gap setting optimizations have been performed according to the procedure described elsewhere [22]. The viscoelastic properties of hydrogels were analyzed under oscillatory shear conditions by applying different procedures. Stress sweep tests $(S S)$ were carried out at constant frequency $(1 \mathrm{~Hz})$ in order to determine the extension of the linear viscoelastic region and the pattern of the nonlinear viscoelastic response. Frequency sweeps $(F S)$ were performed within the linear viscoelastic regime at constant stress (5 $\mathrm{Pa}$ ) in the frequency $(f)$ range of 0.01 to $2 \mathrm{~Hz}$. The mechanical spectra obtained from $F S$ tests were fitted to the generalized Maxwell model consisting of a parallel combination of Maxwell elements and a pure elastic element $\left(g_{\mathrm{e}}\right)$. Accordingly, the dependence of the elastic $\left(G^{\circ}\right)$ and viscous $\left(G^{\prime \prime}\right)$ moduli on pulsation $\omega=2 \pi f$ is given by the following expressions:

$$
\begin{aligned}
G^{\prime} & =g_{e}+\sum_{i=1}^{n_{R}} g_{i} \frac{\left(\lambda_{i} \omega\right)^{2}}{1+\left(\lambda_{i} \omega\right)^{2}} \\
G^{\prime \prime} & =\sum_{i=1}^{n_{R}} g_{i} \frac{\left(\lambda_{i} \omega\right)}{\mid+\left(\lambda_{i} \omega\right)^{2}}
\end{aligned}
$$

where $g_{i}=\eta_{i} / \lambda_{i}, n_{\mathrm{R}}$ is the number of Maxwell elements considered, $g_{\mathrm{i}}, \eta_{\mathrm{i}}$ and $\lambda_{\mathrm{i}}$ represent, respectively, the elastic modulus, the viscosity and the relaxation time of the $\mathrm{i}^{\text {th }}$ Maxwell element. The equilibrium modulus $g_{\mathrm{e}}$ measures the contribution of the purely elastic element. The simultaneous fitting of Eq.(1) and (2) to experimental $G$ and $G$ " data was performed assuming that relaxation times $\left(\lambda_{\mathrm{i}}\right)$ were scaled by a factor 10 [23]. Hence, the parameters of the model were $2+n_{\mathrm{R}}$ (i.e. $\lambda_{1}$, $g_{\text {e }}$ plus $\left.g_{\mathrm{i}}\left(1<i<n_{\mathrm{R}}\right)\right)$. Based on a statistical procedure [24], $n_{\mathrm{R}}$ was selected in order to minimize the product $\chi^{2} *\left(2+n_{\mathrm{R}}\right)$, where $\chi^{2}$ is the sum of the squared errors.

\section{LF-NMR}

The LF-NMR analysis was performed at $25^{\circ} \mathrm{C}$ by means of a Bruker Minispec mq20 (0.47 T, $20 \mathrm{MHz}$, Germany). The determination of the average water protons transverse (spinspin) relaxation time inside the samples $\left(T_{2 \mathrm{~m}}\right)$ was performed according to the CPMG (Carr-Purcell-Meiboom-Gill) [25] sequence $\left\{90^{\circ}\left[-\tau-180^{\circ}-\tau(\text { echo })\right]_{\mathrm{n}}-T_{\mathrm{R}}\right\}$ with a $8.36 \mu$ s wide $90^{\circ}$ pulse, echo time $\tau=250 \mu \mathrm{s}$, and $T_{\mathrm{R}}$ (sequences repetition rate) equal to $5 \mathrm{~s}$. The reason for a so small $\tau$ relies on the $T_{2 \mathrm{~m}}$ dependence on $\tau$ for systems characterised by high $T_{2 \mathrm{~m}}$. Indeed, while $T_{2 \mathrm{~m}}$ is scarcely affected by $\tau$ for $T_{2 \mathrm{~m}}<$ $1500 \mathrm{~ms}$, this is no longer true for higher $T_{2 \mathrm{~m}}$. Accordingly, in order to perform a proper comparison among systems characterised by different $T_{2 \mathrm{~m}}$, we decided to set a small $\tau$ value that returns the best estimation of $T_{2 \mathrm{~m}}$, i.e. the one corresponding to an echo time approaching to zero [26]. The criterion adopted to choose $n$ consisted in ensuring that the final FID intensity corresponded to approximately $1 \%$ of the initial FID intensity. In the light of this acquisition strategy, we verified that it was un-necessary adopting $T_{R}>5$ s. 
Accordingly, $n$ spanned between 400 and 850 . Finally, each FID decay, composed by $n$ points, was repeated 36 times (number of scans). The relaxation times distribution $\left(A_{\mathrm{i}}, T_{2 \mathrm{i}}\right)$ was determined by fitting the FID time decay $\left(I_{\mathrm{s}}\right)$, related to the extinction of the $\mathrm{x}-\mathrm{y}$ component of the magnetization vector, according to its theoretical estimation $I(t)$ [27]:

$I(t)=\sum_{i=1}^{m} A_{\mathrm{i}} \exp \left(-\mathrm{t} / T_{2 \mathrm{i}}\right) \quad T_{2 \mathrm{~m}}=\sum_{i=1}^{m} A_{\mathrm{i}} T_{2 \mathrm{i}} / \sum_{i=1}^{m} A_{\mathrm{i}}$

where $t$ is time, $A_{\mathrm{i}}$ are the pre-exponential factors (dimensionless) proportional to the number of protons relaxing with the relaxation time $T_{2 \mathrm{i}}$ and $T_{2 \mathrm{~m}}$ is the average relaxation time of protons. For a more intuitive meaning, the percentage value of $A_{\mathrm{i}}$ was reported $\left(A_{\mathrm{i} \%}\right)$ :

$A_{\mathrm{i} \%}=100^{*} A_{\mathrm{i}} / \sum_{\mathrm{i}=1}^{\mathrm{m}} A_{\mathrm{i}}$

where $m$ is the number of the relaxation times $\left(T_{2 \mathrm{i}}\right)$ constituting the relaxation time distribution $\left(A_{\mathrm{i}}, T_{2 \mathrm{i}}\right) \cdot m$ was determined by

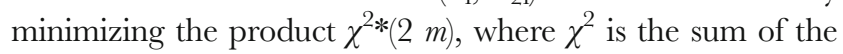
squared errors and $2 m$ represents the number of fitting parameters of eq.(1) [24].

\section{Release Tests}

After crosslinking, hydrogels were washed three times with Hepes 2 buffer ( $0.33 \mathrm{ml}$ for each washing) to collect the superficial liposomes eventually not embedded in the hydrogel. The fluorescence intensity associated to the washing buffer ( $3 \mathrm{X}$ $0.33 \mathrm{~mL}$ ) was assumed as the fluorescent dye at time zero in the release profiles. The release study was performed by immersing the hydrogel membranes $(\sim 1 \mathrm{~mL})$ in $5 \mathrm{~mL}$ Hepes 2 (release environment fluid) at $37^{\circ} \mathrm{C}$. This method was considered the closest to mimic physiological conditions in which either liposomes are absorbed by a target tissue or the release medium is submitted to continuous flow. At fixed times, up to $24 \mathrm{~h}$, the whole release fluid volume - later referred to supernatant - was collected and diluted to a final volume of $6 \mathrm{~mL}$ with fresh buffer. The released samples were stored at $4^{\circ} \mathrm{C}$ until further analyses. Release experiments were performed in triplicate.

Additional release assays were performed to analyse the morphology of released liposomes by cryogenic transmission electron microscopy (cryo-TEM). In these tests, liposomes were prepared with non-fluorescent lipids and the release environment volume was set to $0.5 \mathrm{ml}$ to increase the concentration of the released lipids and improve the chances of identification of the nano-vesicles.

$100 \mu \mathrm{L}$ aliquots of the release medium were collected at defined times and the fluorescence intensity was analysed by means of a Plate reader $\left(\lambda_{\text {abs }}=460 \mathrm{~nm}, \lambda_{\mathrm{em}}=535 \mathrm{~nm}\right.$,Viktor3 Multilabel Plate Reader, Perkin Elmer). Each data point is the average of three repeated measurements. The calibration was performed for each liposome batch, using the same suspension used for the hydrogel under evaluation. The assays used in the calibration were subjected to the same UV irradiation dose used in the preparation of the hydrogel. Indeed, the exposure to UV light produces a pronounced drop of the fluorescence intensity in the liposome suspensions, with a linear dependence with dye concentration and irradiation dose.

\section{Microscopy Analysis}

Two different microscope techniques were used to characterize the hydrogels and the liposomes dispersion within the hydrogel matrix. Transmission electron microscopy (TEM) was employed to visualize the nano-scopic structure of the hydrogel network. Confocal fluorescence (CFM) provided information about the distribution of fluorescent liposomes within the polymer network at different steps: before and after UV irradiation, after spraying of the $\mathrm{CaCl}_{2}$ solution, and after $24 \mathrm{~h}$ from release.

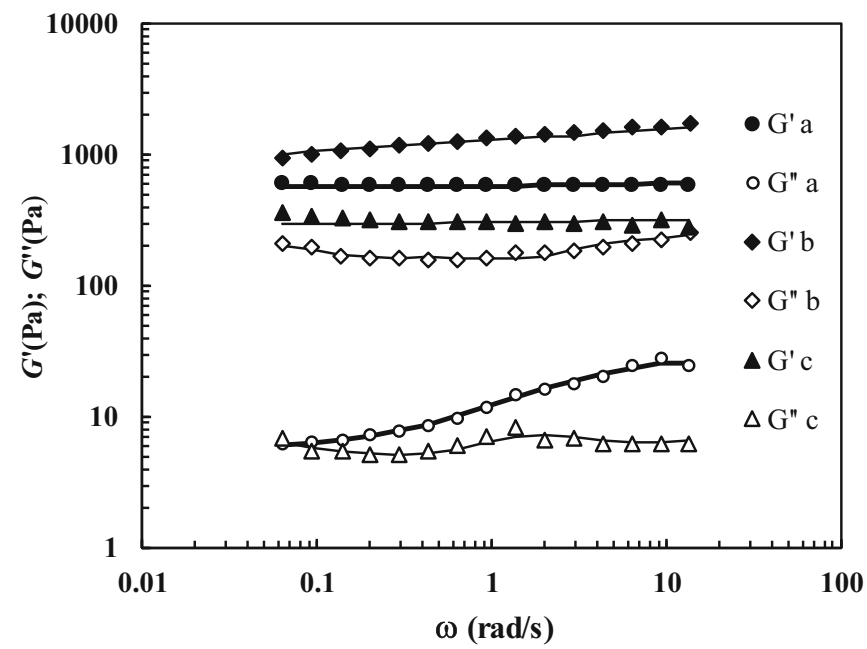


Fig. 2 Effect of temperature $\left(25^{\circ} \mathrm{C}\right.$ $-37^{\circ} \mathrm{C}$ ) on the frequency sweep test (applied stress $\mathrm{T}=5 \mathrm{~Pa}$ ) referring to system a (crosslinking of PVP). G and G" represent the elastic (or storage) and the viscous (or loss) moduli, respectively. Circles refer to $T=25^{\circ} \mathrm{C}\left(\mathrm{Ga} 25^{\circ} \mathrm{C}\right.$ $G$ "a $25^{\circ} \mathrm{C}$ ), while triangles refer to $\mathrm{T}=37^{\circ} \mathrm{C}\left(\mathrm{Ga} 37^{\circ} \mathrm{C}, \mathrm{G}^{\prime \prime} \mathrm{a} 37^{\circ} \mathrm{C}\right)$.

Data standard error, not reported for clarity, are always less than 10\% of the mean.

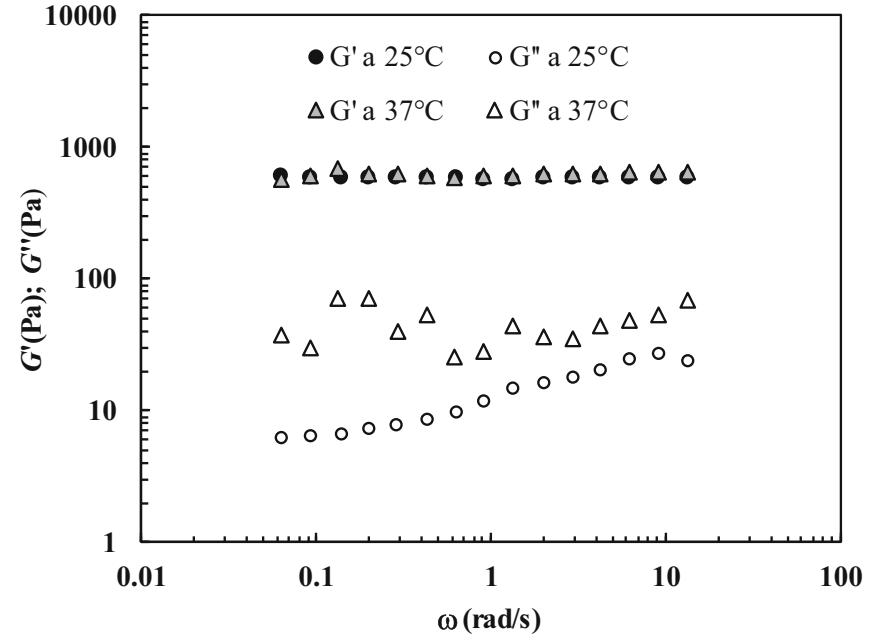

(FEI Vitrobot Mark IV) and were maintained at the cryogenic temperature in liquid nitrogen $\left(\sim-200^{\circ} \mathrm{C}\right)$ until imaging. Cryo-TEM images were taken for the following samples: (i) the native liposome suspension diluted in buffer Hepes 2 (diluted 1:2, $1.25 \mathrm{mg} / \mathrm{ml}$ ); (ii) the dissolution volume sample after $1 \mathrm{~h}$ of release, diluted 1:2; (iii) the release sample from an empty IPN PVP-Alginate hydrogel.

Hydrogel specimens with and without liposomes were examined by TEM (Philips EM 208100 kV). In order to remove the water without damaging the hydrogel structure due to liquid loss, we recurred to a fixative step in glutaraldehyde (GTA). Indeed, GTA, forming covalent bonds with polymeric chains, stabilizes and stiffens the hydrogel structure. Once dehydrated, hydrogels were embedded into epoxy resin that underwent polymerization up to the formation of a solid hard block. Subsequently, the epoxy-block was sliced into thin sections by an ultra-microtome, placed on a copper grid and stained with acetate uranyl; this last chemical produces the highest electron density and image contrast as well as imparting a fine grain to the image due to the high atomic weight of uranium (238).
Fig. 3 Effect of liposomes on the frequency sweep test (applied stress $\mathrm{T}=5 \mathrm{~Pa}, T=37^{\circ} \mathrm{C}$ ) referring to system a (crosslinking of PVP). $G$ and G" represent the elastic (or storage) and the viscous (or loss) moduli, respectively. Triangles refer to system a without liposomes $\left(G \mathrm{a} 37^{\circ} \mathrm{C}, \mathrm{G}\right.$ "a37 $\left.7^{\circ} \mathrm{C}\right)$ while crosses refer to system a loaded by liposomes (G L, G” L). Data standard error, not reported for clarity, are always less than 10\% of the mean.

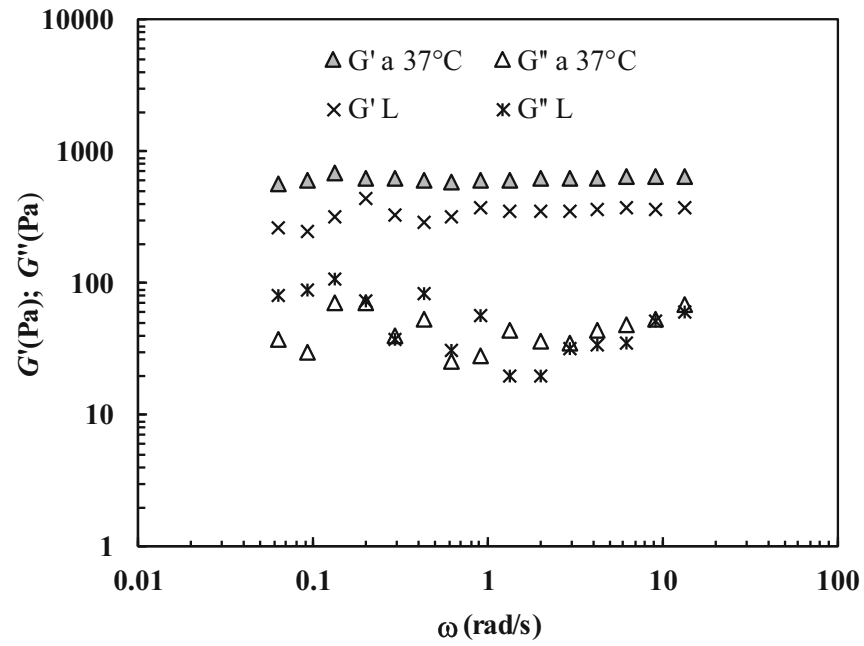


Fig. 4 Water protons transverse (spin-spin) relaxation referring to hydrogels formed by crosslinking of PVP only (system a), alginate only (system b) or PVP and alginate (system c). $t$ is time while $1 / I_{0}$ is the ratio between the value of the magnetization vector $x-y$ component at time $t$ and at time zero. Circles indicate experimental data while continuous lines are eq.(3) best fitting to experimental data. Data standard error, not reported for clarity, are always less than $5 \%$ of the mean.

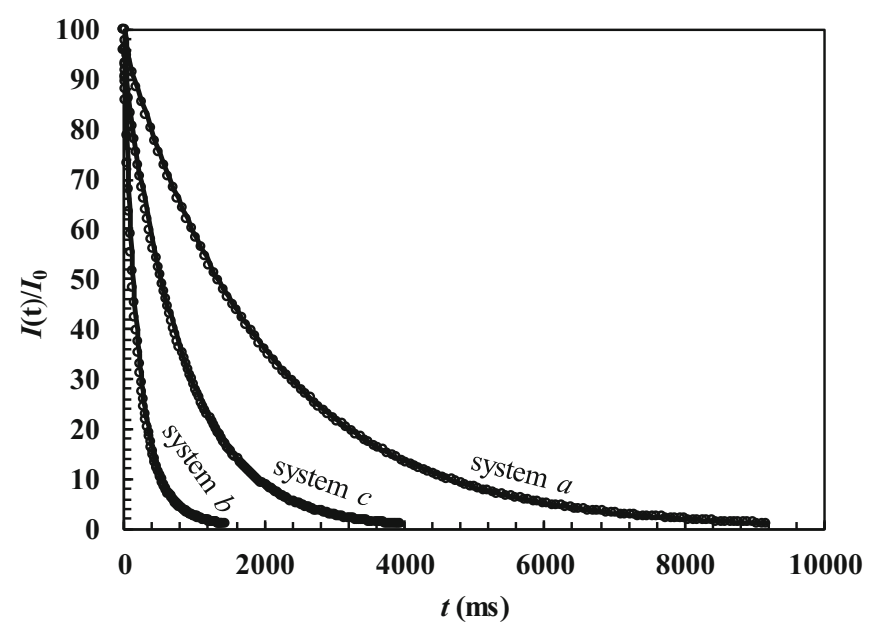

In order to evaluate the effect of temperature and liposomes, frequency sweep characterization was also performed on our reference system (system a) at $37^{\circ} \mathrm{C}$ with and without liposomes as shown, respectively, in Figs. 2 and 3. In particular, Fig. 2 shows that temperature increase does not reflect in any variation of $G^{\prime}$, this meaning that the system structure is substantially unaffected by temperature, while it implies a moderate increase of $G$ ". On the contrary, the addition of liposomes $(\approx 2 \mathrm{mg} / \mathrm{mL})$ implies a small depression of the elastic component $\left(G^{\prime}\right)$, being the viscous one $\left(G^{\prime \prime}\right)$, practically, unaffected. Thus, we should conclude that liposomes presence tends to somehow hinder PVP crosslinking.

LF-NMR characterisation reveals different behaviours for what concerns water protons relaxation related to the three hydrogel specimens considered as shown in Fig. 4. System $b$ (only alginate crosslinked) shows the fastest relaxation followed by system $c$ (both PVP and alginate crosslinked) and, then, system $a$ (crosslinking of PVP only). Analogously, differences among hydrogels arise in relation to liposomes release kinetics as shown in Fig. 5.

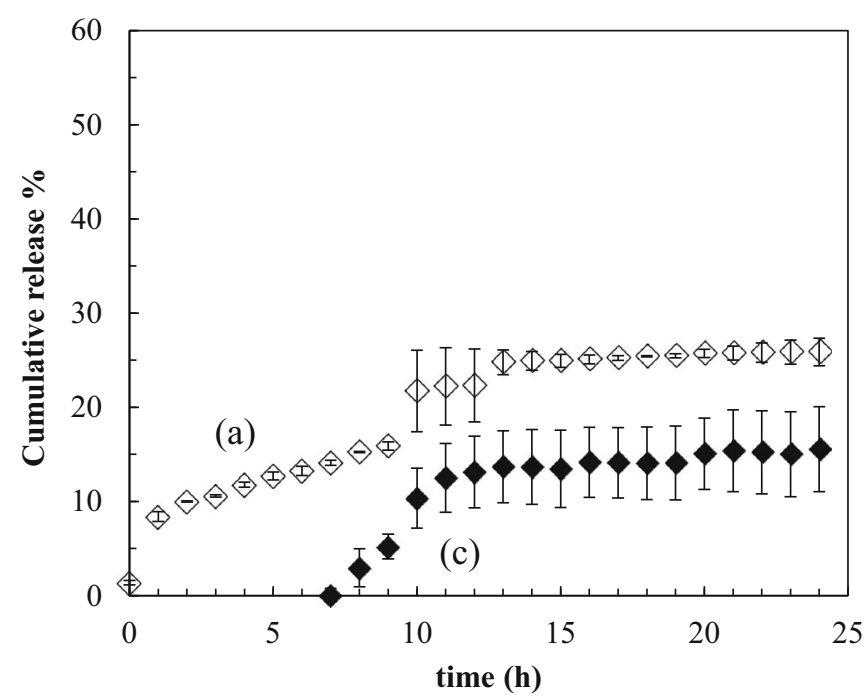


Indeed, system $a$ (crosslinking of PVP only) shows a clearly higher release kinetics than system $c$ (PVP and alginate crosslinking). Liposomes delivery from system $b$ (only alginate crosslinked) exhibited an undetectable fluorescence signal during the whole length of the test. We attributed this lack of signal to multiple causes: (i) a big portion of liposomes are segregated in presumable tight meshes of the alginate network, (ii) the presence of a copious portion of noncrosslinked PVP polymer suspended in the supernatant covered the fluorescence emission of the analyte. TEM images confirm the different structures of our hydrogels. Fig. 6.1, 6.2 and 6.3 depict the TEM images of IPN samples without liposomes, showing the different structures of hydrogel composed by PVP 20\% alone (without alginate; Fig. 6.1), the system $a$ (PVP 20\% + alginate $0.5 \%$ where only PVP underwent crosslinking; Fig. 6.2) and system $c$ (PVP 20\% and alginate 05.\% crosslinking; Fig. 6.3). In the case of Fig. 6.1, only the light grey structures (ribbons) formed by PVP chains are visible (PVP chains are weakly coloured by the contrast agent). On the contrary, as shown in Fig. 6.2, the simple addition of alginate (not crosslinked, strongly coloured by the contrast agent) gives origin to a completely different structure, which hides the original PVP ribbons. Finally, Fig. 6.3 shows the effect of alginate crosslinking on the original PVP network: the original PVP structure (light grey ribbons) connects isolated and globular dark zones. This can be ascribed to crosslinked alginate clusters (heavily coloured by the contrast agent).

CFM provided interesting outcomes about the fate of liposomes once loaded inside the polymeric system. In particular, Fig. 7.1 proves that liposomes are dispersed in the PVP20\%A $0.5 \%$ solution as florescence is substantially uniform all over the scanned area. After PVP crosslinking (Fig. 7.2), fluorescence intensity somehow decreases due to the bleaching effect of UV light inducing PVP reticulation. However, liposomes distribution does not seem to be affected remaining uniform. When also the alginate undergoes crosslinking (Fig. 7.3), it seems that liposomes are clustering into defined micro-domains (shown as bright dots in the image). After $24 \mathrm{~h}$ release, the remaining liposomes in the polymeric IPN are no longer uniformly distributed as shown by the fact that the florescent dye completely disappeared from wide image portions of the scanned area while persisting in some big clusters (Fig. 7.4).

Finally, cryo-TEM was used to analyse the structure of liposomes: while Figs. 8a shows a fresh batch of liposomes right after preparation, Fig. 8b shows liposomes after the release. Both after extrusion and release from the hydrogel, multilamellar vesicles were observed. We imputed this liposome structure to the preparation method. Interestingly, Fig. $8 \mathrm{a}$ and b qualitatively show that released liposomes are characterised by dimensions similar to those of freshly extruded liposomes.
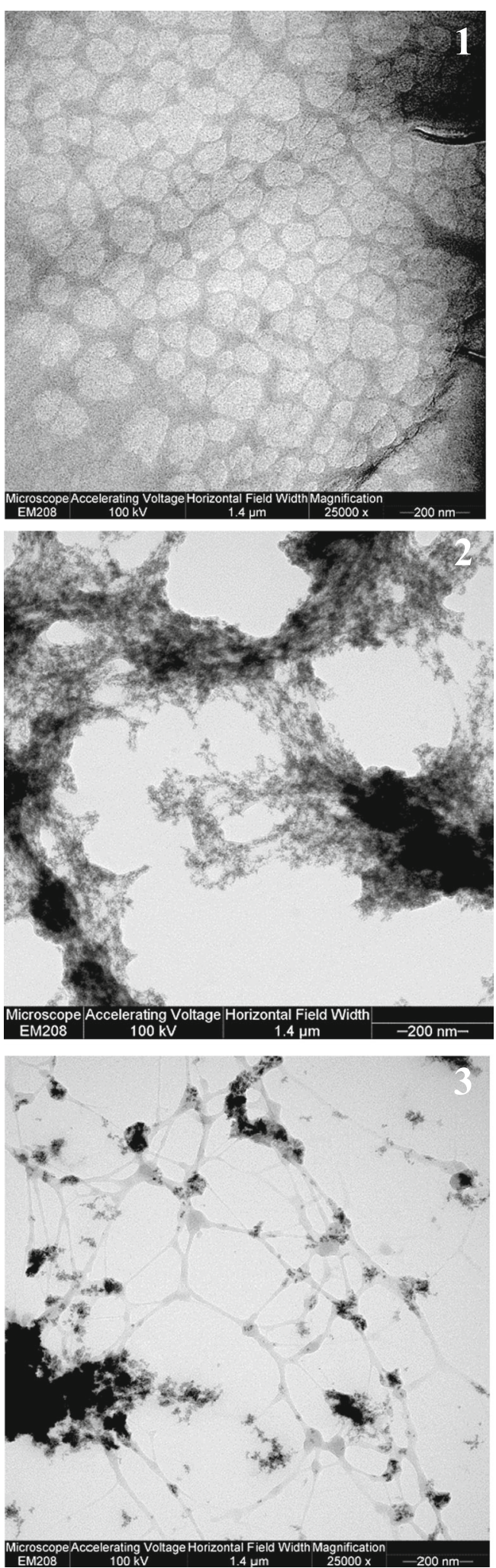

Fig. 6 TEM images of I) a hydrogel formed by crosslinking a PVP 20\% solution (without alginate) 2) system a (PVP 20\%-A0.5\%, crosslinking of PVP only) and 3) system c (PVP 20\%-A0.5\% crosslinking of both PVP and alginate). 
Fig. 7 CFM images of a PVP20\%A0.5\% system loaded with liposomes at different preparation stages: I) polymer solution (before crosslinking) 2) after PVP

crosslinking 3) after PVP and alginate crosslinking 4) after PVP and alginate crosslinking (system c) after $24 \mathrm{~h}$ release.
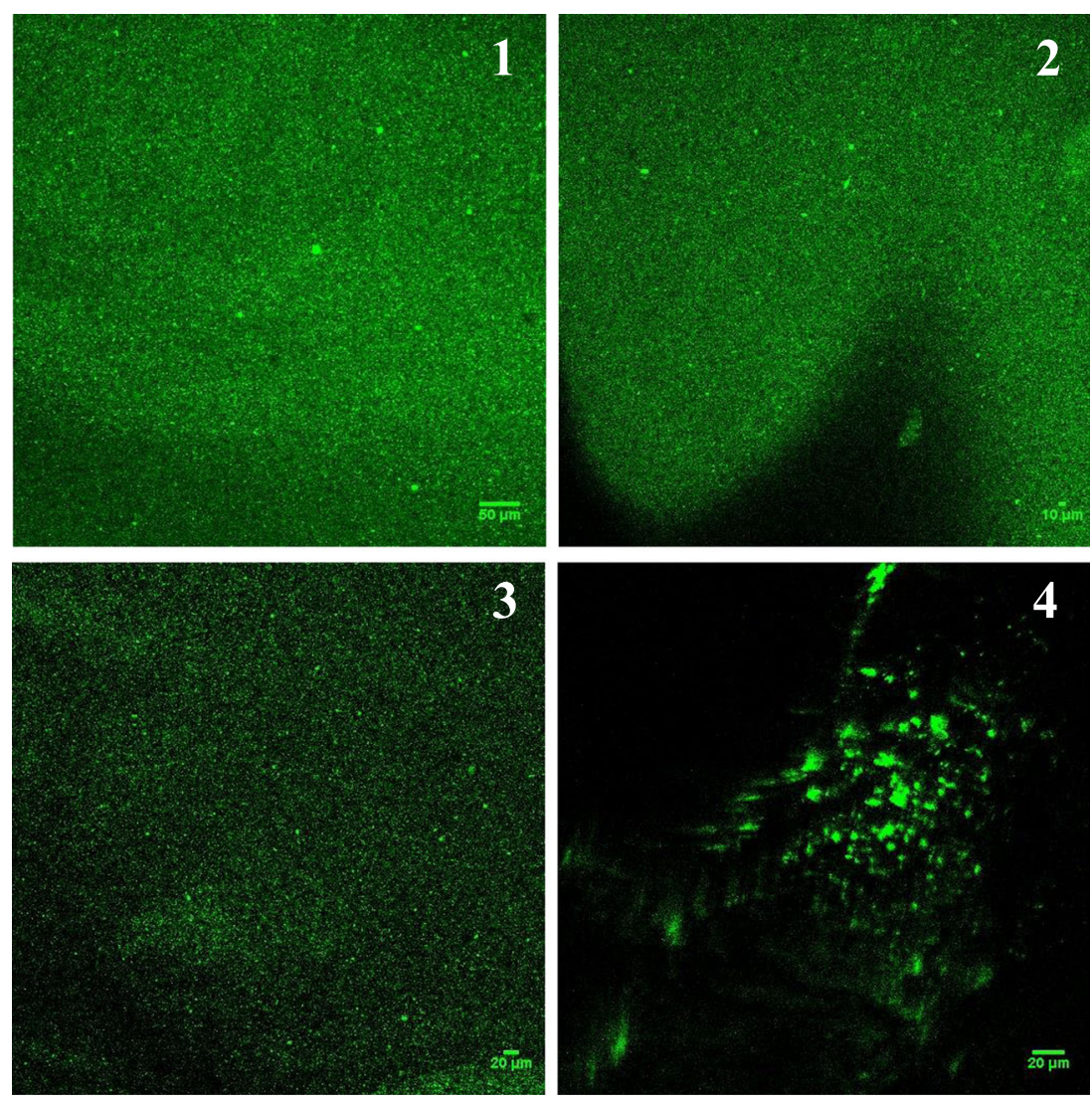

\section{DISCUSSION}

Rheological characterisation reveals that the structures of the three hydrogels are complex as four Maxwell elements plus a purely elastic element are required to properly describe their viscoelastic behaviour (see Table I). In addition, F-test outcomes reveal that the model fitting (eq.(1) and (2)) is always statistically good (see Table I and solid lines in Fig. 1). Interestingly, and unexpectedly, the mechanical strength of the three hydrogels, represented by the shear modulus $G$ (sum of $g_{\text {e }}$ plus four $g_{\mathrm{i}}$ ), is maximum when only alginate undergoes crosslinking (system b), decreases when only PVP is crosslinked (system $a$ ) and assumes the lowest value when both polymers (PVP and alginate) are crosslinked (system $c$ ). This evidence cannot be easily explained as an IPN should be characterized by higher shear modulus than those of the gels where only one of the two polymers undergoes crosslinking.

Indeed, in the case of IPN, crosslink density $\left(\rho_{\mathrm{x}}-\right.$ moles of crosslinks between different chains per unit volume) should be higher and, accordingly to Flory theory [28], this reflects into higher $G$ values

$G=R T \rho_{\mathrm{x}}$

where $R$ is the universal gas constant and $T$ is temperature.

In order to help elucidating this apparent incongruity, the results of LF-NMR can be useful. Indeed, eq.(3) best fitting to relaxation data (solid lines of Fig. 2), statistically good for all hydrogels (see F-test score in Table II), indirectly reveals the presence of different structures for the three hydrogels under study (labelled system $a, b$ and $c$ in Table II).

These hydrogels are characterized by different average relaxation time $\left(\mathcal{T}_{2 \mathrm{~m}}\right)$ and by different distribution of the relaxation times $\left(A_{\mathrm{i} \%}, \mathcal{T}_{2 \mathrm{i}}\right)$. In particular, as system $b$ is characterized by the lowest $\mathcal{T}_{2 \mathrm{~m}}$, Chui and Scherer theories [27, 29] allow concluding that the average polymeric network mesh size $\left(\xi_{\mathrm{m}}\right)$ of this hydrogel is the smallest one among the three hydrogels considered:

$$
\xi_{\mathrm{m}}=\frac{\mathrm{M}}{\left(\frac{1}{T_{2 \mathrm{~m}}}-\frac{1}{T_{2 \mathrm{~b}}}\right) \sqrt{\frac{1-0.58 \phi}{3 \pi \phi}}} \quad \xi_{\mathrm{m}} \propto T_{2 \mathrm{~m}}
$$

where $T_{2 b}$ is the relaxation time of free water protons (i.e. the relaxation time of water protons when polymeric chains are not present), $\phi$ is polymer volume fraction in the hydrogel and $\mathrm{M}$ is the so called relaxivity [27], dimensionally a velocity, that reflects the effect of polymeric chains on the water protons relaxation. Eq.(6) clearly states the proportionality existing between $\xi_{\mathrm{m}}$ and $T_{2 \mathrm{~m}}$. As $\mathrm{M}$ depends also on the chemical properties of the polymeric chains, in the case of interpenetrated networks, it represents an average value between the two polymers. 
Fig. 8 Cryo-TEM picture of liposomes (a) after extrusion, and

(b) released from the system c (both

PVP and alginate crosslinked).
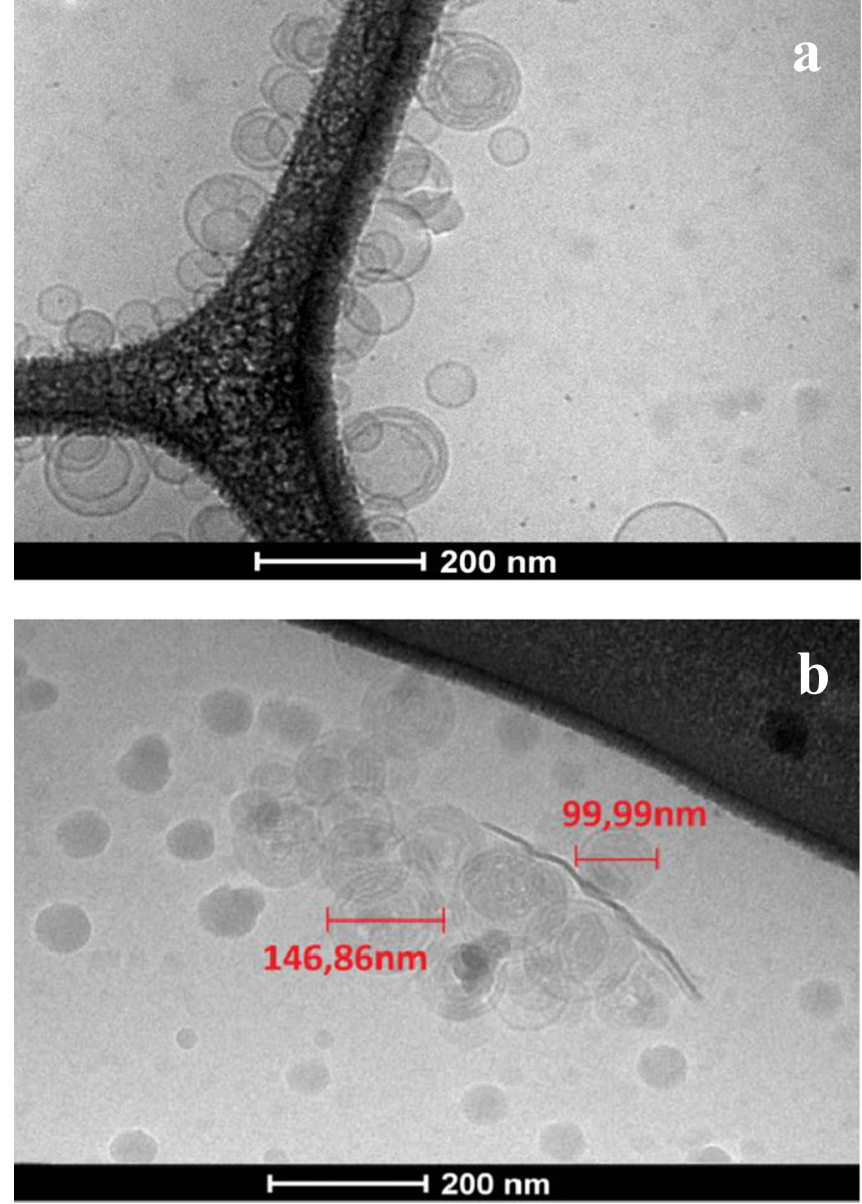

Consequently, for system $b$, LF-NMR and rheology outcomes are concordant as shown by the fact that this hydrogel is associated with the smallest $T_{2 \mathrm{~m}}$ and the highest $G$ (see Tables I and II).

On the contrary, rheology and LF-NMR are apparently discordant for what concerns the properties of system $a$ and $c$. Indeed, while rheology indicates that system $c$ is weaker than system a $(G$ decreases, see Table I), LF-NMR indicates that

Table I Values of eq.(I) and (2) Fitting Parameters Referring to System a (pvp Crosslinking Only), b (Alginate Crosslinking Only) and c (pvp and Alginate Crosslinking). G Indicates the Shear Modulus, (Equal to One Third of Young Modulus, E, in the Case of Incompressible Materials as our Hydrogels), while $F$ Indicates the Statistical F-test Score (Accomplished for all Systems)

\begin{tabular}{llll}
\hline & System $a$ & System b & System c \\
\hline$\lambda_{1}(\mathrm{~s})$ & $0.0635 \pm 0.005$ & $0.0195 \pm 0.010$ & $0.0589 \pm 0.0085$ \\
$g_{e}(\mathrm{~Pa})$ & $554.6 \pm 6.7$ & $785.4 \pm 117.0$ & $279.5 \pm 6.8$ \\
$g_{1}(\mathrm{~Pa})$ & $48.5 \pm 1.7$ & $597.4 \pm 72.0$ & $10.4 \pm 0.8$ \\
$g_{2}(\mathrm{~Pa})$ & $18.3 \pm 1.1$ & $264.2 \pm 57.0$ & $11.1 \pm 0.7$ \\
$g_{3}(\mathrm{~Pa})$ & $7.4 \pm 0.8$ & $196.4 \pm 41.5$ & $4.9 \pm 0.9$ \\
$g_{4}(\mathrm{~Pa})$ & $11.8 \pm 2.1$ & $356.5 \pm 78.6$ & $17.1 \pm 3.0$ \\
$G(\mathrm{~Pa})=g_{e}+\sum_{i=1}^{n_{R} g_{i}}$ & $640.6 \pm 7.5$ & $2200.0 \pm 173.5$ & $323.1 \pm 7.5$ \\
$F(5,25,0.95)<$ & 1885 & & 472 \\
\hline
\end{tabular}


Table II Equation (3) Fitting Parameters Referring to System a (PVP Crosslinking Only), b (Alginate Crosslinking Only) and c (PVP and Alginate Crosslinking). $T_{2 m}$ is the Average Spin-Spin Relaxation Time, $\left(A_{i} \%, T_{2 i}\right)$ Represent the Relaxation Time Distribution while $F$ Indicates the Statistical F-Test Score

\begin{tabular}{llll}
\hline & System $a$ & System $b$ & System c \\
\hline$T_{2 \mathrm{~m}}(\mathrm{~ms})$ & 1600 & 217 & 749 \\
$T_{21}(\mathrm{~ms})$ & $2101 \pm 10$ & $438 \pm 1.0$ & $856 \pm 0.1$ \\
$T_{22}(\mathrm{~ms})$ & $1646 \pm 60$ & $171 \pm 0.3$ & $10 \pm 0.1$ \\
$T_{23}(\mathrm{~ms})$ & $18 \pm 1.2$ & $14 \pm 0.2$ & - \\
$A_{1 \%}(-)$ & $67.2 \pm 3$ & $23.1 \pm 0.2$ & $87.3 \pm 0.1$ \\
$A_{2 \%}(-)$ & $11.2 \pm 3$ & $66.8 \pm 0.1$ & $12.7 \pm 0.1$ \\
$A_{3 \%}(-)$ & $21.6 \pm 1.5$ & $10.1 \pm 0.1$ & - \\
& $F(5,844,0.95)<6.9 * 10^{7}$ & $F(5,394,0.95)<3.4^{*} 10^{7}$ & $F(5,696,0.95)<1.2^{*} 10^{8}$ \\
\hline
\end{tabular}

system $c$ is characterized by lower $T_{2 \mathrm{~m}}$ with respect to that of system $a$ (see Table II). A possible spatial organisation able to account for the rheology and the LF-NMR outcomes on system $c$ implies the existence of an interconnected PVP network pervading the whole gel volume and presenting isolated clusters of crosslinked alginate. In this way, alginate clusters would be responsible for the $T_{2 \mathrm{~m}}$ reduction as they entrap water protons inside their small meshes (see eq.(6)). At the same time, they cannot affect hydrogel mechanical strength $(G)$ as they do not constitute an interconnected network but detached islands embedded in the PVP network. Assuming that clusters formation weakens the pre-existing PVP network (alginate crosslinking implies a considerable shrinking process that can disrupt or damage the PVP network fibres in contact or inside clusters), the mechanical weakening ( $G$ decrease) of system $c$ with respect to system $a$ can be explained. Figures 6.1, 6.2 and 6.3 seem to support the proposed structure. Indeed, Fig. 6.2, referring to system $a$, shows alginate fibres coating PVP fibres that are not visible. Upon alginate crosslinking (system $c$ ), the structure of the polymeric network modifies and the appearance of alginate clusters (dark spots) connected each other by PVP fibres (grey ribbons, Fig. 6.1) becomes evident (Fig. 6.3).

The existence of the proposed structure is also reinforced by the release tests (see Fig. 5) showing how liposomes release kinetics is different for system $a$ and $c$. Indeed, not only liposomes are more quickly released from system $a$, but system $c$ shows a significant delay $(7 \mathrm{~h})$ before the release starts. In addition, the final concentration plateau referred to system $c$ is lower than the one of system $a$, which indicates a limited liposomes delivery. This evidence might be connected to the presence of alginate clusters which reduces liposomes mobility and implies liposomes a remarkable entrapment. Interestingly, while Fig. 7.1, 2 and 3 show an almost uniform distribution of liposomes inside system $c$, Fig. 7.4 shows how, after $24 \mathrm{~h}$ release, a considerable liposomes fraction is still present but only in some detached regions most likely represented by alginates clusters. Finally, it is interesting to point out that liposomes incorporation and release has not detrimental effects on liposome integrity (Fig. 8), this being relevant for the use of the studied polymeric matrices as liposome delivery materials.

\section{CONCLUSIONS}

This paper demonstrates the potentiality of the combined use of rheology and LF-NMR for the characterisation of very complex structures such as interpenetrated hydrogels. Indeed, the outcomes of these characterizations have been proved by other independent tests such as release tests, TEM and fluorescent microscopy images. In our opinion, this group of techniques proved to be very useful to design and optimise IPN hydrogels devoted to the release of active agents. Indeed, a detailed knowledge of nanostructure is mandatory to properly design and optimize drug delivery system based on polymers such as IPN. This is particularly true in the case of the release of active agents that need to be embedded inside liposomes or on their surface in order to protect them till they reach the site action as it happens in the case of the fragile Nucleic Acid Based Drugs [31-37].

\section{ACKNOWLEDGMENTS AND DISCLOSURES}

This work was supported by the Italian Ministry of Education (PRIN 2010-11 (20109PLMH2)), by Fondo di Ateneo FRA 2016 - Trieste University, BIOFLUO project, POR FESR 2014-2020, FVG, Italy. The authors would like to acknowledge the Danish National Research Foundation (DNRF122) and Villum Fonden (Grant No. 9301) for Intelligent Drug Delivery and Sensing Using Microcontainers and Nanomechanics (IDUN). Authors would like to acknowledge Prof. Leticia Hosta-Rigau and Dr. Maria Godoy Gallardo for the interesting discussions and the valuable inputs given to the investigation.

\section{REFERENCES}

1. Peppas NA, Bures P, Leobandung W, Ichikawa H. Hydrogels in pharmaceutical formulations. Eur J Pharm Biopharm. 2000;50:2746.

2. Hennink WE, van Nostrum CF. Novel crosslinking methods to design hydrogels. Adv Drug Deliv Rev. 2012;64:223-36.

3. Hoffman AS. Hydrogels for biomedical applications. Adv Drug Deliv Rev. 2012;64:8-23. 
4. Dai W, Barbari T. Hydrogel membranes with mesh size asymmetry based on the gradient crosslinking of poly(vinyl alcohol). J Memb Sci. 1999;156:67-79.

5. Peppas NA, Berner RE. Proposed method of intracopdal injection and gelation of poly (vinyl alcohol) solution in vocal cords: polymer considerations. Biomaterials. 1980;1:158-62.

6. Kuijpers A, van Wachem P, van Luyn MJ, Engbers GH, Krijgsveld $\mathrm{J}$, Zaat SA, et al. In vivo and in vitro release of lysozyme from crosslinked gelatin hydrogels: a model system for the delivery of antibacterial proteins from prosthetic heart valves. J Control Release. 2000;67:323-36.

7. Sperinde JJ, Griffith LG. Synthesis and characterization of enzymatically-cross-linked poly(ethylene glycol) hydrogels. Macromolecules. 1997;30:5255-64.

8. Jeun JP, Jeon YK, Nho YC, Kang PH. Effects of gamma irradiation on the thermal and mechanical properties of chitosan/PVA nanofibrous mats. J Ind Eng Chem. 2009;15:430-3.

9. Jabbari E, Nozari S. Swelling behavior of acrylic acid hydrogels prepared by $\gamma$-radiation crosslinking of polyacrylic acid in aqueous solution. Eur Polym J. 2000;36:2685-92.

10. Aiji Z, Othman I, Rosiak JM. Production of hydrogel wound dressings using gamma radiation. Nucl Instruments Methods Phys Res Sect B Beam Interact with Mater Atoms. 2005;229:375-80.

11. Yoshii F, Darwis D, Mitomo H, Makuuchi K. Crosslinking of poly( $\varepsilon$-caprolactone) by radiation technique and its biodegradability. Radiat Phys Chem. 2000;57:417-20.

12. Gorna K, Gogolewski S. The effect of gamma radiation on molecular stability and mechanical properties of biodegradable polyurethanes for medical applications. Polym Degrad Stab. 2003;79:46574.

13. Decker G. Kinetic study and new applications of UV radiation curing. Macromol Rapid Commun. 2002;23:1067-93.

14. Almeida JF, Ferreira P, Lopes A, Gil MH. Photocrosslinkable biodegradable responsive hydrogels as drug delivery systems. Int J Biol Macromol. 201 1;49:948-54.

15. Lopérgolo LC, Lugão AB, Catalani LH. Direct UV photocrosslinking of poly(N-vinyl-2-pyrrolidone) (PVP) to produce hydrogels. Polymer (Guildf). 2003;44:6217-22.

16. Fechine GJM, Barros JAG, Catalani LH. Poly(N-vinyl-2-pyrrolidone) hydrogel production by ultraviolet radiation: new methodologies to accelerate crosslinking. Polymer (Guildf). 2004;45:47059 .

17. Marizza P, Abrami M, Keller SS, Posocco P, Laurini E, Goswami $\mathrm{K}$, et al. Synthesis and characterization of UV photocrosslinkable hydrogels with poly(Nvinyl-2-pyrrolidone): determination of the network mesh size distribution. Journal of Polymeric Materials and Polymeric Biomaterials. 2016;65:516-25.

18. Bochicchio S, Dalmoro A, Barba AA, Grassi G, Lamberti G. Liposomes as siRNA delivery vectors. Curr Drug Metab. 2014;15:882-92.

19. Grassi M, Cavallaro G, Scirè S, Scaggiante B, Dapas B, Farra R, et al. Current strategies to improve the efficacy and the delivery of nucleic acid based drugs. Current signal transduction Therapy. 2010;5:92-120.

20. Barba AA, Lamberti G, Sardo C, Dapas B, Abrami M, Grassi M, et al. Novel lipid and polymeric materials as delivery systems for nucleic acid based drugs. Curr Drug Metab. 2015;16:427-52.
21. Scarabel L, Perrone F, Garziera M, Farra R, Grassi M, Musiani F, et al. Strategies to optimize siRNA delivery to hepatocellular carcinoma cells. Expert opinion on Drug Delivery. 2017;14:797-810.

22. Kuijpers AJ, Engbers GHM, Feijen J, De Smedt SC, Meyvis TKL, Demeester J, et al. Characterization of the network structure of Carbodiimide cross-linked gelatin gels. Macromolecules. 1999;32: 3325-34.

23. Lapasin R, Pricl S. Rheology of industrial polysaccharides. Blackie Academic and Professional, Chapman and Hall, Glasgow, UK: Theory and Applications; 1995.

24. Draper NR, Smith H. Applied regression analysis. John Wiley \& Sons, Inc., New York, 1966.

25. Meiboom S, Gill D. Modified SpinEcho method for measuring nuclear relaxation times. Rev Sci Instrum. 1958;29:688-91.

26. Abrami M, Ascenzioni F, Di Domenico EG, Maschio M, Ventura A, Confalonieri M, Di Gioia S, Conese M, Dapas B, Grassi G, Grassi M. A novel approach based on low field nuclear magnetic resonance for the detection of the pathological components of sputum in cystic fibrosis patients. Magn. Reson. Med. 2018 in press, 22 AUG 2017, DOI: https://doi.org/10.1002/mrm.26876.

27. Chui M, Phillips R. Measurement of the porous microstructure of hydrogels by nuclear magnetic resonance. J Colloid Interface Sci. 1995; 174:336-44.

28. Flory PJ. Principles of polymer chemistry. Ithaca, USA: Cornell University, Press; 1953.

29. Scherer GW. Hydraulic radius and mesh size of gels. J Sol-Gel Sci Technol. 1994;1:285-91.

30. Schurz J, Rheology of polymer solutions of the network type. Prog Polym Sci, 1991;16:1991, 1-53, 1.

31. Farra R, Dapas B, Pozzato G, Scaggiante B, Agostini F, Zennaro C, et al. Effects of E2F1-cyclin E1-E2 circuit down regulation in hepatocellular carcinoma cells. Dig Liver Dis. 201 1;43:1006-14.

32. Grassi G, Schneider A, Engel S, Kandolf R, Kuhn A. Hammerhead ribozymes targeted against cyclin E and E2F1 cooperate to down-regulate coronary smooth muscle cell proliferation. Journal of Gene medicine. 2005;7:1223-34.

33. Grassi G, Marini JC. Ribozymes: structure, function and potential therapy for dominant genetic disorders. Ann Med. 1996;28:499510.

34. Grassi G, Dawson P, Kandolf R, Guarnieri G, Grassi M. Therapeutic potential of hammerhead ribozymes in the treatment of hyper-proliferative diseases. Curr Pharm Biotechnol. 2004;5: 369-86.

35. Bochicchio S, Dapas B, Russo I, Ciacci C, Piazza O, De Smedt S, et al. In vitro and ex vivo delivery of tailored siRNA-nanoliposomes for E2F1 silencing as a potential therapy for colorectal cancer. Int J Pharm. 2017;525:377-87.

36. Farra R, Scaggiante B, Guerra C, Pozzato G, Grassi G, Zanconati $\mathrm{F}$, et al. Dissecting the role of the elongation factor $1 \mathrm{a}$ isoforms in hepatocellular carcinoma cells by liposome-mediated delivery of siRNAs. Int J Pharm. 2017;525:367-76.

37. Grassi G, Scaggiante B, Dapas B, Farra R, Tonon F, Lamberti G, et al. Therapeutic potential of nucleic acid-based drugs in coronary hyper-proliferative vascular diseases. Curr Med Chem. 2013;20: 3515-8. 